\section{Thomas Harriot}

\begin{abstract}
Astronome et mathématicien anglais, Thomas Harriot est surtout connu pour ses travaux en algèbre moderne, pour son étude de la théorie de la réfraction et pour avoir produit les premières esquisses de la Lune observée au travers d'une lunette astronomique en juillet 1609 (donc quelques mois avant les travaux historiques de Galilée).
\end{abstract}

Riad HAIDAR, haidar@onera.fr

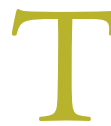
homas Harriot nait à Oxford, probablement en 1560. On sait si peu de choses sur son enfance qu'on ignore la date exacte de sa naissance et que son nom a subi plusieurs mutations au cours des siècles : on le retrouve orthographié sous la forme de Harriott, Hariot, ou encore Heriot. Les seuls indices que l'on ait trouvés sont issus des registres de la prestigieuse et élitiste université d'Oxford: on y découvre que Harriot y est admis le vendredi 20 décembre 1577, à l'âge de 17 ans (d'où l'année estimée de sa naissance), qu'il est né dans le Oxfordshire, et qu'il est d'extraction modeste (son père est qualifié de plébéein). Néanmoins, son inscription à Oxford indique que sa famille était manifestement aisée.

\section{Le cartographe}

À Oxford, Harriot étudie au St Mary's Hall. Il en sort diplômé ès arts en 1582, et s'installe à Londres. En 1583, il entre au service de Sir Walter Raleigh, courtisan en vue à la cour d'Elisabeth I et puissant armateur londonien. Employé d'abord comme précepteur en mathématiques, il étend très vite le champ de ses prérogatives et utilise ses connaissances en astronomie pour établir des cartes de navigation et conseiller la conception des navires de son protecteur. Enfin, ayant gagné sa confiance, il devient le comptable de Raleigh, en charge de l'obtention de fonds pour financer les futures expéditions.

À cette époque, Harriott se lie avec deux amérindiens, Manteo et Wanchese, des Algonquins originaires de Caroline du Nord, fraîchement arrivés en Angleterre. Il met au point un alphabet phonétique pour transcrire leur langage... Ces préparatifs linguistiques se révéleront d'une importance capitale lors de l'expédition, dirigée par Richard Grenville et financée par Raleigh, qui l'emmène en Amérique en 1585. C'est au cours de ce voyage, où il fait office d'expert et conseiller scientifique, qu'il doit déterminer la façon la plus économique d'empiler des boulets de canon - problème

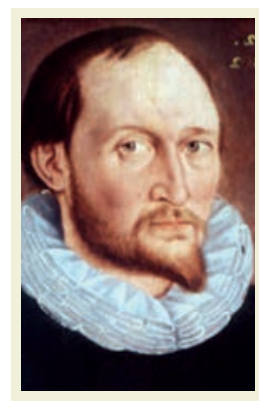

\section{PRINCIPALES DATES}

1560 - naissance à Oxford (Angleterre)

Décembre 1577 Admission à Oxford

1585

Expédition en Caroline du Nord

Juillet 1601

Découverte de la loi des sinus

Juillet 1609 Dessin des cratères de la lune

2 Juillet 1621 - Décès à Londres (Angleterre)

Portrait supposé de Thomas Harriot.

qu'il extrapolera à la théorie des empilements de sphères, puis à la théorie atomistique sur laquelle il aura des échanges fertiles avec Johannes Kepler. Kepler a l'intuition géniale que l'on obtient l'empilement le plus dense si, pour chaque couche, les centres des sphères sont au-dessus des centres des trous de la couche inférieure, une conjecture qui ne sera confirmée qu'en 1998 par la démonstration numérique de Thomas Hales de l'université du Michigan. À cause de ces réflexions trop originales pour l'époque, Harriot est ouvertement accusé d'athéisme.

Dans le Nouveau Monde, Harriot fait partie de la petite colonie de Roanoke, une île de l'archipel côtier des Outer Banks, en Caroline du Nord, et y perfectionne sa connaissance de l'algonquin. Il explore et cartographie la baie de Chesapeake. En 1586, il rentre en Angleterre avec l'expédition de Francis Drake, de retour de Saint-Domingue. L'année suivante, il rédige une grammaire de l'algonquin

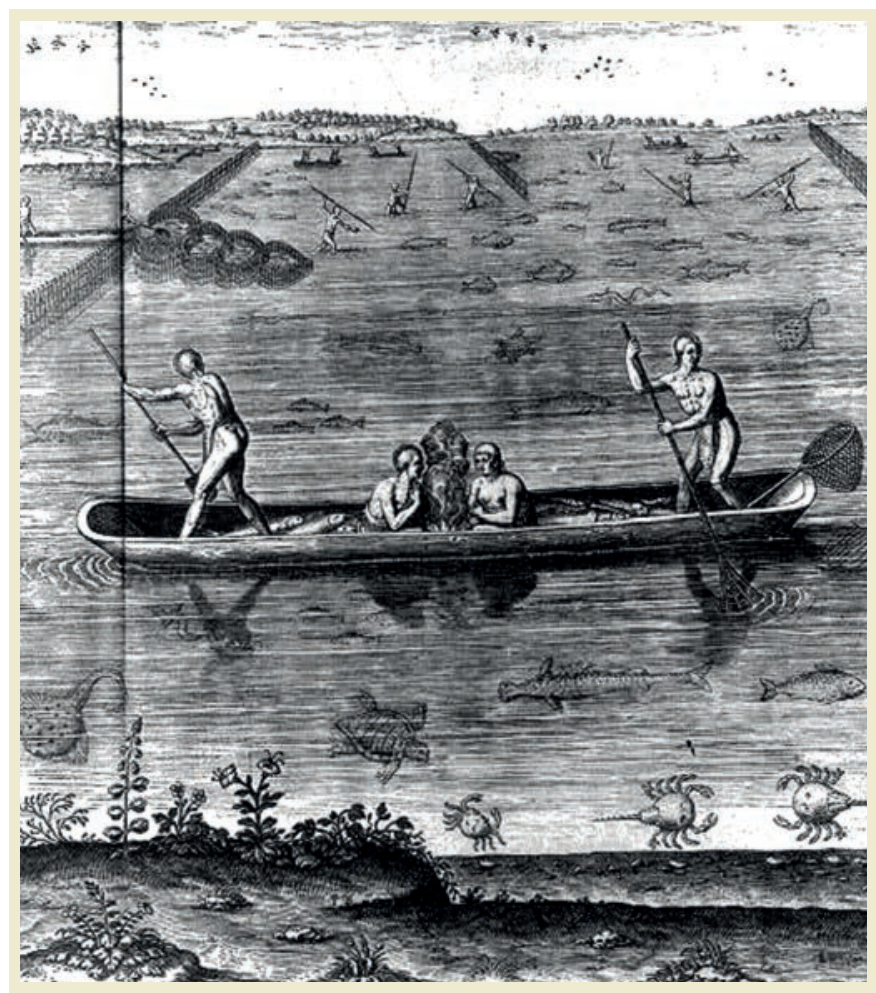

La baie de Pamlico, dessinée par John White (1590).

Harriot y passe une année à étudier les mœurs des Algonquins. 
et publie ses comptes-rendus de voyage, un livre qui aura une grande influence sur plusieurs générations d'explorateurs et colons anglais.

\section{Travaux scientifiques}

Au cours des années 1590, Harriot s'intéresse à l'algèbre, qu'il étudie d'abord dans les écrits de John Dee, avant de découvrir les ouvrages de François Viète - et de s'enthousiasmer à leur lecture. En 1595, il devient l'un des protégés de Henry Percy, comte du Northumberland. Dès 1597, Percy lui ouvre les portes de sa propriété de Syon House, près de Kew dans les environs de Londres, et l'autorise à y installer un laboratoire où il peut mener ses expériences scientifiques. Il lui offre également une terre près de Durham, et Harriot intègre ainsi la bourgeoisie enviée des propriétaires terriens.

À Syon, Thomas Harriot mène ses premiers travaux en optique. Sa découverte la plus remarquable, quoique non publiée (comme d'ailleurs le reste de son œuvre scientifique), est la loi des sinus pour la réfraction de la lumière qui, d'après les carnets qu'il a laissés, date de juillet 1601 - soit 36 ans avant sa première publication par Descartes. Il étudie également le problème dit d'Alhazen sur la réflexion catoptrique : il s'agit d'un tracé de lignes partant de deux points à l'intérieur d'un cercle réunis en un point situé sur la circonférence et faisant des angles égaux avec la perpendiculaire à ce point.

La gouvernance de Syon House est confiée à un parent du comte, Thomas Percy, connu pour avoir participé à la Conspiration des poudres ${ }^{1}$. En 1605, accusés de complicité avec Thomas Percy, les protecteurs de Harriot, Walter Raleigh et Henry Percy, tombent en disgrâce et sont incarcérés dans la tour de Londres. Harriot lui-même est brièvement emprisonné, avant d'être blanchi... Henri Percy, qui ne recouvrera sa liberté qu' après seize années de geôle, maintient néanmoins son soutien financier à Harriot.
En 1607, Harriot s' intéresse à l' astronomie lors du passage de la comète de Halley. Il est, avec son ami astronome William Lower, l'un des premiers à utiliser une lunette télescopique pour des observations astronomiques : il étudie et dessine les cratères lunaires le 26 juillet 1609 , soit quelques mois avant Galilée. On lui doit également l'étude de la balistique des boulets de canon, dont il établit que la trajectoire est toujours parabolique.

\section{Ultima verbae}

En 1616, Thomas Harriot montre les premiers signes d'un cancer des lèvres, dont il note lui-même l'évolution à partir de 1618. Ce cancer progresse en cancer de la peau, et l'emporte le 2 juillet 1621 à Londres, dans la maison de son ami Thomas Buckner, sur Threadneedle Street près du Royal Exchange.

Son œuvre, quoique d'une importance majeure, ne joue pourtant qu'une faible influence dans le développement des sciences au cours des siècles qui suivent - sans doute parce qu'il n'a pas publié ses résultats. Le seul livre qui nous soit parvenu, Artis Analyticae Praxis ad Aequationes Algebraicas Resolvendas, a été édité et publié en 1631, soit 10 ans après sa mort.

\section{RÉFÉRENCE \\ [1] John W. Shirley, Thomas Harriot: A Biography (Oxford, 1983).}

1 La Conspiration des poudres (en anglais Gunpowder Plot) est une ten-

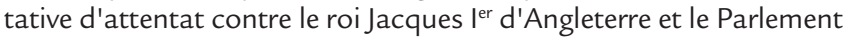
anglais par un groupe de catholiques provinciaux anglais conduits par sir Robert Catesby. Le projet prévoit de faire sauter la Chambre des communes au cours de la cérémonie d'ouverture du Parlement du 5 novembre 1605. L'attentat doit être le prélude à une révolte populaire dans les Midlands au cours de laquelle la fille du roi, la princesse Élisabeth, alors âgée de neuf ans, sera installée sur le trône d'un État catholique.

\section{Filtres Interférentiels}

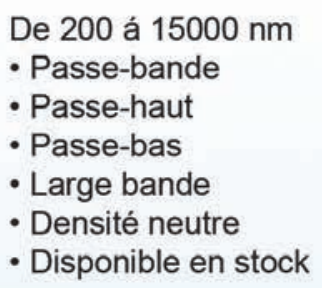

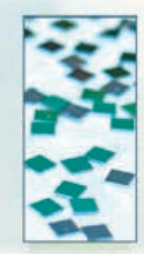

UK (parle francais): sales.uk@spectrogon.com·Tel +44 1592770000

Sweden (headquarters): sales.se@spectrogon.com· Tel +46 86382800 US: sales.us@spectrogon.com·Tel+19733311191

De 150 á $2000 \mathrm{~nm}$

- Compression d'impulsion

- Télècom

- Accordabilité spectrale

- Monochromateurs

- Spectroscopie

- Disponible en stock 